\title{
Escalas para medir las fases de autorregulación del aprendizaje en estudiantes de secundaria
}

\author{
Fabiola Sáez-Delgado $^{1^{\star}}$, Javier Mella-Norambuena ${ }^{2}$, Yaranay López-Angulo ${ }^{3}$ y Verónica León-Ron ${ }^{4}$ \\ (1) Universidad Católica de la Santísima Concepción, Facultad de Educación, Departamento Fundamentos de la \\ Pedagogía, Concepción-Chile (correo-e: fsaez@ucsc.cl) \\ (2) Universidad Técnica Federico Santa María, Departamento de Ciencias, Concepción, Chile \\ (correo-e: javier.mellan@usm.cl) \\ (3) Universidad Santo Tomás, Facultad de Ciencias Sociales y Comunicaciones, Escuela de Psicología, Concepción, \\ Chile. (correo-e: yara13190@gmail.com) \\ (4) Universidad Técnica del Norte, Facultad de Educación, Ciencia y Tecnología, Ibarra, Ecuador. \\ (correo-e: mvleon@utn.edu.ec)
}

Recibido Ago. 19, 2020; Aceptado Oct. 21, 2020; Versión final Dic. 20, 2020, Publicado Abr. 2021

\begin{abstract}
Resumen
El objetivo de esta investigación fue diseñar y estimar las propiedades psicométricas de escalas que miden las tres fases del proceso de autorregulación del aprendizaje. El diseño fue instrumental y participaron 438 estudiantes de educación secundaria de Ecuador. Las escalas se diseñaron a partir de la revisión de la literatura, validadas por jueces expertos y entrevistas cognitivas. Además, se realizaron análisis factoriales exploratorios, confirmatorios y de confiablidad. Los resultados indicaron adecuados índices de validez y confiabilidad; quedó conformada por 53 ítems representados en 5 escalas: 1) Estrategias de disposición al estudio, 2) autoeficacia para la disposición al estudio, 3) estrategias cognitivas, metacognitivas, y búsqueda de ayuda, 4) atribuciones causales, y 5) autoevaluación. Se concluye que las escalas son útiles para medir el proceso de aprendizaje autorregulado completo o procesos específicos, tanto para investigadores que diseñan intervenciones como para los profesores que buscan promoverla.

Palabras clave: aprendizaje autorregulado; escala de medida; educación secundaria; propiedades psicométricas
\end{abstract}

\section{Scales to measure self-regulated learning phases in secondary school students}

\begin{abstract}
The objective of this research study was to design and estimate the psychometric properties of scales that measure the three phases of the self-regulated learning process. The design was instrumental and 438 secondary education students from Ecuador participated. The scales were designed based on a literature review and were validated by expert judges and by performing cognitive interviews. In addition, exploratory, confirmatory, and reliability factor tests were performed. The results showed adequate validity and reliability indices. There were 53 items represented in five scales: 1) strategies for disposition to study, 2) self-efficacy for disposition to study, 3) cognitive and metacognitive strategies and seeking help, 4) causal attributions, and 5) self-assessment. It is concluded that the scales designed here are useful for measuring the entire or specific part of the self-regulated learning process by researchers who design interventions and by teachers who seek to promote it.
\end{abstract}




\section{INTRODUCCIÓN}

El aprendizaje autorregulado (en adelante AAR) es un sistema cognitivo motivacional del aprendizaje. Abarca una visión holística del desarrollo de habilidades, conocimientos, motivaciones, creencias y comportamientos por parte de los estudiantes en sus experiencias académicas, que van, desde la planificación de una tarea académica hasta la persistencia frente a la frustración y ajuste respectivo de estrategias para lograr un resultado exitoso. El constructo de AAR representa formas de aprendizaje académico autónomo, permanente y efectivo. Desde la revisión teórica de la literatura, la aplicación del concepto de AAR es denominada autorregulación del aprendizaje (en adelante ARA), la cual, en su definición clásica, se describe como los pensamientos, sentimientos y acciones autogeneradas por los estudiantes y que son planeadas, intencionadas y adaptadas cíclicamente para el logro de objetivos personales del estudio y aprendizaje (Zimmerman, 2000).

Existen varios modelos de ARA que enfatizan diferentes construcciones y mecanismos, aunque hay consenso en conceptualizarla como un proceso cíclico y compuesto por diferentes fases y subprocesos (Panadero, 2017b). Esta conclusión de homologación fue planteada por una revisión teórica que comparó cinco modelos de ARA (Puustinen y Pulkkinen, 2001) y recientemente confirmada por otra revisión que ratifica esta clasificación, señalando tres fases comunes: (a) disposición, que consiste en actividades relevantes para el análisis de tareas, fijación de objetivos y planificación; (b) desempeño, se realiza la tarea y se completa con el progreso del desempeño monitoreado; y (c) evaluación, incluye la evaluación y la reflexión de los resultados (Panadero, 2017b).

Los estudiantes autorregulados se distinguen por su conciencia de cómo estrategias específicas pueden influir en los resultados de su aprendizaje y la disposición de estos a emplearlas en función de lograr sus objetivos académicos. Demuestran tener las habilidades cognitivas y metacognitivas, así como las creencias y actitudes motivadoras necesarias para planear, comprender, supervisar y dirigir su propio aprendizaje. Estos estudiantes son proactivos en sus esfuerzos para aprender; además, son conscientes de sus fortalezas y limitaciones académicas (Zimmerman, 2000). Los estudiantes de alto rendimiento, en comparación con los de bajo y promedio, muestran un enfoque de aprendizaje más estratégico y adaptativo durante todas las fases de su proceso de aprendizaje. Se orientan y planifican de manera más estratégica y efectiva, combinan diferentes estrategias cognitivas y adoptan la autoevaluación para regular su proceso de aprendizaje (DiFrancesca et al., 2016).

Desde la revisión empírica de la literatura, la autorregulación ha capturado la atención de los investigadores pues se considera el núcleo de muchos problemas identificados en los mecanismos de estudio y desempeña un papel central en explicar el logro académico (Yan, 2020). Actualmente, se considera que el AAR abarca muchos de los predictores más sólidos del logro y, a su vez, ha sido identificada como fundamental para prevenir el fracaso escolar (Fernandez-Rio et al., 2017). Específicamente en estudiantes de secundaria, un metaanálisis exploró cómo el AAR influye en el rendimiento académico, sus resultados indican que los procesos metacognitivos y cognitivos del AAR actúan como variables que influyen directamente en el desempeño académico (Dent y Koenka, 2016). Por su parte, Annalakshmi (2019) informó que la autorregulación fue un predictor significativo de la resiliencia y el rendimiento académico en estudiantes de familias rurales de bajos ingresos. En la misma línea de investigación, se encontró relaciones estadísticamente positivas entre el AAR y la motivación intrínseca, extrínseca, el valor de la tarea, el control de las creencias de aprendizaje, la autoeficacia y el rendimiento académico (El-Adl y Alkharusi, 2020). Por tanto, la importancia del AAR en el desempeño de los estudiantes secundarios, ha sido evidenciada en diferentes asignaturas como matemáticas (Lawrence y Saileella, 2019) y química (Şen y Yilmaz, 2016). Sin embargo, aun cuando se ha demostrado que la ARA es importante, esta se ha encontrado en niveles medios (Cleary y Kitsantas, 2017) e incluso bajos, considerándose en ambos casos, inadecuados en grupos de estudiantes de nivel secundario (Fernandez-Rio et al., 2017). Los estudiantes que carecen de estrategias de autorregulación podrían conducir a resultados de aprendizaje ineficaces y consecuentemente no alcanzar las exigencias académicas.

Por lo anterior, se requiere contar con instrumentos de medición de los niveles de ARA de los estudiantes, que permitan identificar aquellos componentes que se encuentran en un nivel de desarrollo deficiente para fomentar y promover oportunamente su desarrollo en contextos académicos. Sin embargo, la medición de este constructo no ha sido una cuestión resuelta ni fácil; esto responde a lo complejo y multidimensional de su definición y por tantos componentes que la conforman. Para alcanzar una definición operativa de la ARA, es importante distinguir entre las fases y los procesos específicos que componen cada una de estas, guiadas por los principales modelos y conceptualizaciones de la ARA, para tener el potencial de corroborar las afirmaciones teóricas y empíricas de la práctica educativa. La medición de este constructo complejo, multidimensional, que integra diferentes habilidades, estrategias, creencias, se transforma en un desafío. La investigación reciente se ha planteado por objetivo descubrir los procesos de AAR de los estudiantes de forma específica y no general (Cerezo et al., 2020). 
Aunque se identifican instrumentos en nivel de secundaria para medir la ARA, estos presentan una desventaja y restricción importante, señaladas por los mismos autores en sus trabajos, la cual corresponde a la medición de, únicamente, algunos componentes de la ARA, es decir, no se ha encontrado un instrumento o conjunto de escalas disponibles que mida íntegramente las fases y componentes de este constructo. Así, por ejemplo, al analizar algunos instrumentos, unos se focalizan en un componente específico de la ARA como el Cuestionario de motivación y estrategias de aprendizaje (MSLQ), que se enfoca en componentes principalmente motivacionales (Pintrich et al., 1991), mientras otros miden la ARA como un proceso general, no distinguiendo sus diferentes componentes, como por ejemplo el Inventario de estrategias de autorregulación (Cleary y Callan, 2014). Considerando las restricciones actuales que se observan en las herramientas disponibles para medición de la ARA en estudiantes de secundaria, es una necesidad diseñar, validar y estudiar la estructura factorial de un instrumento que refleje la medición del proceso completo de la ARA y que considere las tres fases comunes de los distintos modelos de la ARA, cuestión que ha quedado demostrada en dos revisiones sistemáticas recientes que se han enfocado en describir los instrumentos de ARA disponibles para estudiantes de distintos niveles académicos (León-Ron et al., 2020) y otra específicamente en secundaria (López-Angulo et al., 2020).

El objetivo de este estudio fue diseñar y estimar las propiedades psicométricas de escalas que miden las tres fases de ARA en estudiantes secundarios ecuatorianos. Eso permitirá responder a la actual necesidad de contar con una herramienta de medición válida y confiable del constructo de AAR en estudiantes secundarios. A su vez, facilitará la medición de la efectividad de intervenciones que tengan por propósito mejorar los procesos de autorregulación en este nivel académico con mediciones antes y después de la implementación de la intervención.

\section{MÉTODO}

Este es un estudio cuantitativo con diseño instrumental (Ato et al., 2013) que consideró tres etapas principales: Diseño y validación; análisis factorial exploratorio y análisis factorial confirmatorio.

\section{Participantes}

El acceso a los participantes fue por conveniencia, en el mes de noviembre de 2019. La muestra final incluida en esta investigación fue de 438 estudiantes de Educación Secundaria (199 mujeres y 239 hombres), el promedio de edad fue de 13,53 años $(D E=1.56)$. Para los análisis factoriales se dividió la muestra quedando 200 estudiantes en el análisis factorial exploratorio y 238 en el análisis factorial confirmatorio. Los establecimientos educativos participantes fueron dos públicos y uno privado; todos de la provincia de Imbabura, ciudad de Ibarra, Ecuador.

\section{Instrumento}

Las escalas incluidas para la medición de procesos de autorregulación fueron diseñadas por los autores de este estudio. En primer lugar, se consideró la homologación de fases de los modelos existentes de ARA, es decir, se delimitaron 3 fases de este meta constructo: disposición, desempeño y evaluación basado en el modelo de (Zimmerman, 2000). En segundo lugar, se seleccionaron ítems de la revisión de instrumentos disponibles en la literatura, estos fueron: cuestionario de autorregulación del aprendizaje online (Jansen et al., 2017); la escala de atribuciones causales (Sáez et al., 2019); la escala de autorreporte de autorregulación del aprendizaje (Toering et al., 2012); el cuestionario de autoeficacia para la autorregulación de la disposición estudio (Sáez et al., 2018); escala de autorregulación académica (Akhtar y Mahmood, 2013); y el Inventario de estrategias de autorregulación (Cleary, 2006). En tercer lugar, se crearon ítems en base a la necesidad de aproximarnos al AAR como un constructo multidimensional y vinculado a la teoría especializada en consistencia con las tres fases y sus respectivos procesos (Zimmerman, 2000).

Se diseñaron 5 escalas independientes. La fase de disposición y/o planificación quedó representada por dos escalas: (a) Escala de estrategias de disposición al estudio con 11 ítems y la (b) escala de autoeficacia para la disposición al estudio con 7 ítems. La fase de desempeño quedó constituida de una sola escala denominada: (c) escala de estrategias cognitivas y metacognitivas del estudio con 21 ítems. Finalmente, la fase de evaluación quedó constituida por dos escalas: (d) escala de atribuciones causales con 12 ítems y la (e) escala de autoevaluación del estudio y aprendizaje con 19 ítems. Las escalas son unidimensionales, a excepción de la escala de atribuciones causales que tiene dos subescalas: atribuciones causales de fracaso relacionadas al esfuerzo y la habilidad y, atribuciones causales de fracaso relacionadas a factores externos. En total, las escalas diseñadas inicialmente quedaron compuesta por 70 ítems, distribuidos en tres fases.

\section{Procedimiento}

Para la confección de cada escala específica de procesos de autorregulación, una primera etapa contempló la revisión de las recomendaciones encontradas en revisiones sistemáticas de la literatura que han tenido por 
propósito identificar y caracterizar instrumentos para la medir la ARA en distintos niveles académicos (LeónRon et al., 2020; López-Angulo et al., 2020). Adicionalmente, se identificaron artículos psicométricos específicos sobre ARA. Los resultados de esta revisión de la literatura permitieron la selección de ítems para cada una de las 5 escalas de procesos de autorregulación, de tal forma que dé cuenta del complejo y multidimensional proceso de ARA. Los ítems seleccionados que estaban en un idioma distinto al español fueron sometidos a un proceso de traducción y retro traducción.

Una vez obtenida la primera propuesta de cada escala, esta fue enviada a 5 jueces expertos (Urrutia et al., 2015), tres internacionales (Chile, Venezuela y Cuba) y dos nacionales (Ecuador), para dar cuenta de la evidencia de validez de contenido. Estos expertos fueron seleccionados intencionalmente debiendo cumplir con criterios específicos de selección (grado de Doctor en Psicología, Doctor en Educación o Doctor en Metodología; experiencia en el diseño de instrumentos, con al menos una publicación en el tema de la ARA). Se les envió a los jueces un correo electrónico en el cual se les informó el objetivo de la investigación y se les pidió su colaboración para participar del proceso de validación. Todos los jueces a los cuales se les escribió respondieron favorablemente a la solicitud. Este correo adjuntaba un formulario que debían completar para dar su valoración sobre cada uno de los ítems que integraban las escalas. La valoración consistió en: cumple o no cumple el ítem respecto de los criterios de claridad, pertinencia, suficiencia y coherencia de estos con la dimensión. Además, se les pidió realizar observaciones o sugerencias de la redacción de los ítems si lo estimaban pertinente.

En este proceso se eliminaron 4 ítems; además, la recomendación más frecuente de los expertos tuvo relación con la mejora de la claridad de los ítems dado el contexto cultural, por tanto, se reescribieron 5 ítems. El índice de Kappa fue de .92; lo que indica un grado adecuado de acuerdo entre los jueces. Luego de la incorporación de las recomendaciones de los expertos, se desarrollaron 7 entrevistas cognitivas a estudiantes de educación secundaria, seleccionados intencionalmente para la identificación de problemas de comprensión en los ítems y dar cuenta de la evidencia de validez de respuesta de la escala (Urrutia et al., 2015). Se solicitó a los estudiantes comentarios respecto de la claridad de las instrucciones, el formato de respuesta, comprensión de los ítems de cada una de las escalas. Este proceso de entrevista cognitiva evidenció que los estudiantes comprendían las instrucciones; sin embargo, dos ítems, en más de 3 estudiantes tuvieron dificultades, por tanto, se decidió eliminarlos. Finalmente, los autores de este trabajo realizaron una última revisión para identificar inconsistencias, incoherencias y dificultades, pero no se detectaron, por tanto, no se eliminaron más ítems (ver figura 1).

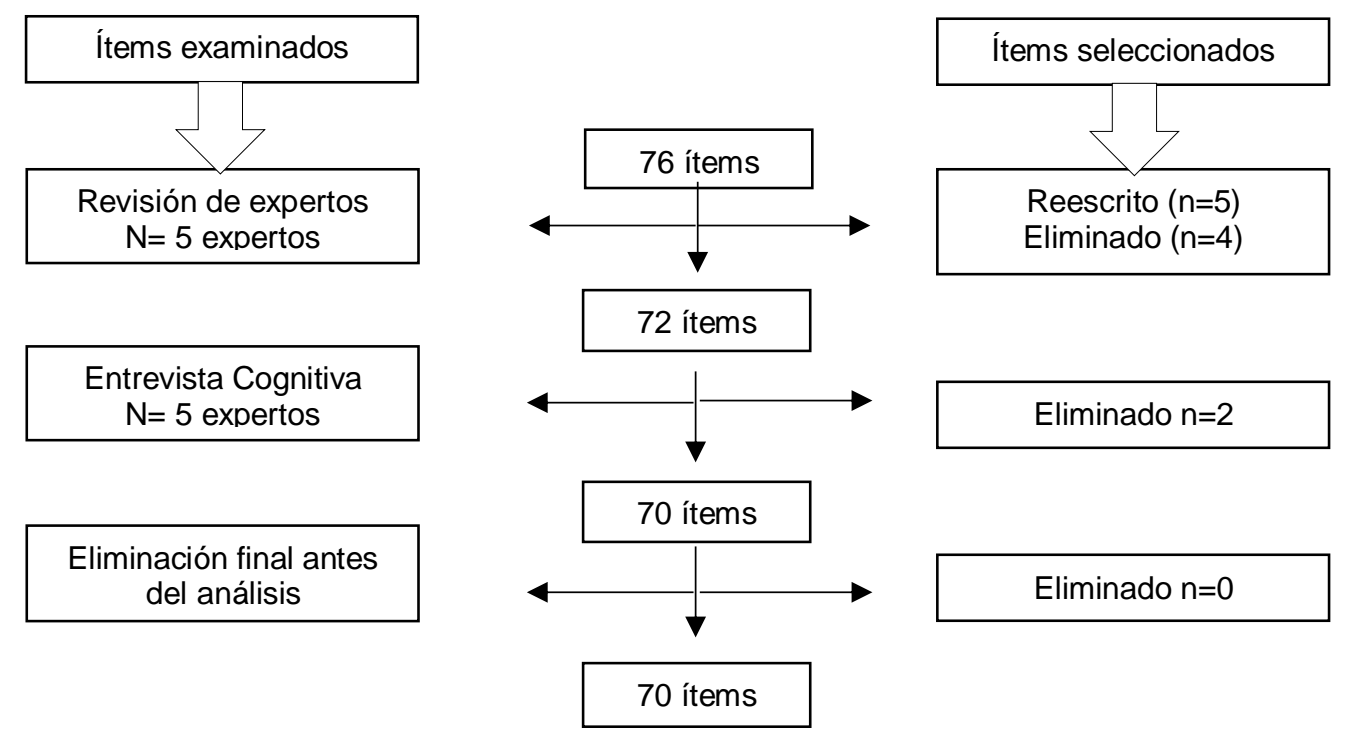

Figura 1. Proceso de validación y selección de ítems de las escalas de ARA

\section{Consideraciones éticas}

Este estudio fue sometido a la aprobación del comité de Ética de la Universidad Católica de la Santísima Concepción en Chile y la Universidad Técnica del Norte en Ecuador. Además, se presentó el estudio a las autoridades de los establecimientos educativos de la Ciudad de Ibarra, en Ecuador y aquellos que lo respaldaron, permitieron la aplicación de consentimientos informados, socialización y autorización por parte de los padres y, posteriormente, se aplicaron las escalas a los estudiantes. 


\section{Análisis de datos}

Para cada una de las escalas se efectuó análisis factorial exploratorio (AFE), este análisis se basó en la matriz de correlaciones policóricas, dado que los ítems están en nivel ordinal (Holgado-Tello et al., 2010). Un primer paso fue revisar el índice KMO (Kaiser, Meyer y Olkin) y la prueba de esfericidad de Bartlett con el objetivo de analizar la existencia de una posible estructura factorial. El segundo paso fue determinar el número de factores usando el análisis paralelo. En el caso de los instrumentos de más de un factor, se identificaron cargas cruzadas de los ítems, es decir, cargaban en más de un factor, por lo que se utilizaron rotaciones en base a matrices target para evaluar si los ítems se estaban distribuyendo en coherencia con la estructura teórica propuesta del instrumento. Además, para evaluar la estructura factorial se realizaron análisis factoriales confirmatorios (AFC) a las cinco escalas. Se consideraron los índices de ajuste sugeridos en la literatura: RMSEA $\leq .07$; CFI y TLI > 0.92 y SRMR < 0.08 (Hair et al., 2014) y chi cuadrado (Kline, 2011). Finalmente, se calculó la consistencia interna de cada escala con el Alfa de Cronbach y Omega de Mcdonald. Para todos los análisis se usó el software RStudio versión 4.0.

\section{RESULTADOS}

A continuación, se describen los resultados del análisis factorial exploratorio, confirmatorio y confiabilidad de las escalas.

\section{Análisis factorial exploratorio}

La prueba de esfericidad de Bartlett fue significativa, $X^{\wedge} 2(55)=984.607, p<.001$, y el $\mathrm{KMO}$ fue de .85 , lo que evidencia una matriz de correlaciones apta para el análisis factorial (Izquierdo et al., 2014). Luego, se procedió a realizar el análisis paralelo de Horn en base a 5000 remuestras con método de ejes principales, los resultados muestran, para algunos casos, 1 factor y para otros, dos factores, lo cual es consistente con la estructura teórica de las escalas que integran la batería. Un primer factor se observó para las escalas de estrategias para la disposición al estudio, autoeficacia para la disposición al estudio y autoevaluación del estudio y aprendizaje y dos factores para las escalas de atribuciones causales y ejecución, éstos explican en todos los casos al menos un $55 \%$ de la varianza. De los 70 ítems iniciales de todas las escalas quedaron 53 en total, es decir se eliminaron 17 ítems.

Tabla 1. Cargas factoriales de las escalas unidimensionales de disposición al estudio, autoeficacia para la disposición y autoevaluación del proceso de ARA.

\begin{tabular}{|c|c|c|c|c|c|}
\hline $\begin{array}{l}N^{\circ} \text { ítems en } \\
\text { escala final }\end{array}$ & $\begin{array}{l}\text { Carga en } \\
\text { Factor } 1\end{array}$ & $\begin{array}{l}N^{\circ} \text { items en } \\
\text { escala final }\end{array}$ & $\begin{array}{l}\text { Carga en } \\
\text { Factor } 1\end{array}$ & $\begin{array}{c}N^{\circ} \text { ítems en escala } \\
\text { final }\end{array}$ & $\begin{array}{l}\text { Carga en } \\
\text { Factor } 1\end{array}$ \\
\hline \multicolumn{2}{|c|}{$\begin{array}{l}\text { Escala de Estrategias de } \\
\text { Disposición al Estudio (DE) }\end{array}$} & \multicolumn{2}{|c|}{$\begin{array}{c}\text { Escala de Autoeficacia para la } \\
\text { DE }\end{array}$} & \multicolumn{2}{|c|}{$\begin{array}{c}\text { Escala de autoevaluación del estudio } \\
\text { y aprendizaje }\end{array}$} \\
\hline P1. Ítem 3 & 0,56 & P2. Ítem 4 & 0,72 & P5. Ítem 8 & 0,81 \\
\hline P1. Ítem 5 & 0,55 & P2. Ítem 2 & 0,68 & P5. Ítem 4 & 0,75 \\
\hline P1. Ítem 6 & 0,60 & P2. Ítem 3 & 0,66 & P5. Ítem 1 & 0,75 \\
\hline P1. Ítem 1 & 0,60 & P2. Ítem 1 & 0,63 & P5. Ítem 6 & 0,75 \\
\hline P1. Ítem 7 & 0,50 & P2. Ítem 5 & 0,58 & P5. Ítem 10 & 0,75 \\
\hline P1. Ítem 2 & 0,58 & P2. Ítem 6 & 0,49 & P5. Ítem 2 & 0,73 \\
\hline P1. Ítem 4 & 0,52 & P2. Ítem 7 & 0,45 & P5. Ítem 12 & 0,74 \\
\hline & & & & P5. Ítem 14 & 0,70 \\
\hline & & & & P5. Ítem 5 & 0,69 \\
\hline & & & & P5. Ítem 7 & 0,69 \\
\hline & & & & P5. Ítem 9 & 0,66 \\
\hline & & & & P5. Ítem 11 & 0,61 \\
\hline & & & & P5. Ítem 3 & 0,59 \\
\hline & & & & P5. Ítem 13 & 0,50 \\
\hline
\end{tabular}

Específicamente, en las escalas unifactoriales se eliminaron 4 ítems de la escala de estrategias de disposición al estudio; y 5 ítems de la escala de autoevaluación del estudio y el aprendizaje. En todos los casos, la eliminación de ítems fue por cargas factoriales inferiores a .40 quedando sólo aquellos ítems con carga superiores a este valor (ver Tabla 1). De las escalas de dos factores, se eliminaron 4 ítems de atribuciones causales y 4 ítems de la escala de ejecución. En estas dos escalas de más de un factor, la eliminación de ítems se debió a la carga cruzada mostrada entre los dos factores que conformaban la escala, y a cargas 
factoriales inferiores a 0.4. De manera iterativa, se procedió a la eliminación de cada ítem a medida que iban presentando cargas cruzadas o bajas comunalidades totales, hasta que finalmente se llegó a una lista de ítems en cada escala y en el caso de aquellas con más de un factor, con cargas óptimas en el respectivo factor. En la tabla 2 se presentan los ítems agrupados a cada factor del instrumento con sus respectivas cargas.

Es importante destacar que, originalmente, se esperaba que la escala de estrategias cognitivas y metacognitivas, se separara en dos factores según la teoría, una para las estrategias cognitivas y otra para las metacognitivas; sin embargo, el resultado del AFE mostró que, si bien se dividió en dos factores, una escala, con sólo tres ítems, se agrupó en un factor que se llamó "búsqueda de ayuda", mientras los demás ítems se agruparon en el factor dos, y este integra estrategias cognitivas y metacognitivas, por lo que lleva este nombre (Ver tabla 2).

Tabla 2. Cargas factoriales de las escalas de disposición al estudio, autoeficacia para la disposición y autoevaluación del proceso de ARA.

\begin{tabular}{|c|c|c|c|c|c|c|c|}
\hline $\begin{array}{l}N^{\circ} \text { ítems en } \\
\text { escala final }\end{array}$ & $\begin{array}{l}\text { Carga en } \\
\text { Factor } 1\end{array}$ & $\begin{array}{l}\text { Carga en } \\
\text { Factor } 2\end{array}$ & $\begin{array}{l}\text { Factor } \\
\text { Teórico }\end{array}$ & $\begin{array}{l}N^{\circ} \text { items en escala } \\
\quad \text { final }\end{array}$ & $\begin{array}{l}\text { Carga en } \\
\text { Factor } 1\end{array}$ & $\begin{array}{l}\text { Carga en } \\
\text { Factor } 2\end{array}$ & $\begin{array}{l}\text { Factor } \\
\text { Teórico }\end{array}$ \\
\hline \multicolumn{4}{|c|}{ Escala de Atribuciones causales } & \multicolumn{4}{|c|}{$\begin{array}{l}\text { Escala de Ejecución: monitoreo, cognitivas, y búsqueda de } \\
\text { ayuda }\end{array}$} \\
\hline P4. Ítem 4 & 0,62 & 0,15 & 1 & P3. Ítem 12 & 0,64 & 0,15 & 1 \\
\hline P4. Ítem 1 & 0,61 & 0,10 & 1 & P3. Ítem 3 & 0,65 & 0,20 & 1 \\
\hline P4. Ítem 6 & 0,59 & 0,23 & 1 & P3. Ítem 11 & 0,65 & 0,27 & 1 \\
\hline P4. Ítem 3 & 0,57 & 0,17 & 1 & P3. Ítem 14 & 0,65 & 0,32 & 1 \\
\hline P4. Ítem 7 & 0,55 & 0,19 & 1 & P3. Ítem 2 & 0,62 & 0,19 & 1 \\
\hline P4. Ítem 5 & 0,17 & 0,80 & 2 & P3. Ítem 13 & 0,57 & 0,15 & 1 \\
\hline P4. Ítem 2 & 0,20 & 0,74 & 2 & P3. Ítem 10 & 0,59 & 0,23 & 1 \\
\hline \multirow[t]{10}{*}{ P4. Ítem 8} & 0,19 & 0,62 & 2 & P3. Ítem 7 & 0,58 & 0,27 & 1 \\
\hline & & & & P3. Ítem 8 & 0,56 & 0,22 & 1 \\
\hline & & & & P3. Ítem 1 & 0,55 & 0,13 & 1 \\
\hline & & & & P3. Ítem 5 & 0,54 & 0,32 & 1 \\
\hline & & & & P3. Ítem 17 & 0,53 & 0,18 & 1 \\
\hline & & & & P3. Ítem 9 & 0,51 & 0,05 & 1 \\
\hline & & & & P3. Ítem 15 & 0,51 & 0,28 & 1 \\
\hline & & & & P3. Ítem 6 & 0,21 & 0,60 & 2 \\
\hline & & & & P3. Ítem 4 & 0,09 & 0,61 & 2 \\
\hline & & & & P3. Ítem 16 & 0,22 & 0,54 & 2 \\
\hline
\end{tabular}

\section{Análisis factorial confirmatorio}

Los resultados observados en la tabla 3 indican que todos los índices de ajuste cumplieron con los criterios adecuados sugeridos en la literatura RMSEA $\leq .07$; CFI y TLI > 0.92 y SRMR < 0.08 (Hair et al., 2014). Si bien el chi cuadrado es significativo, es conocida la sensibilidad que tiene esta prueba a tamaños de muestras grandes (>200) (Kline, 2011). En la tabla 3 se muestra Df correspondientes a los grados de libertad del modelo; el RMSEA correspondiente al Error Medio Cuadrático de Aproximación; el Intervalo de confianza del 90\% para RMSEA; el SRMR correspondiente al Error cuadrático medio de aproximación estandarizado; el CFI correspondiente al Índice de Ajuste Comparativo; el TLI correspondiente al índice de Tucker-Lewis; y con asterisco aquellos valores significativos $(p<.005)$.

Tabla 3. Estimaciones del modelo de AFC de las 5 escalas de ARA

\begin{tabular}{|l|l|c|c|c|c|c|c|c|}
\hline & \multicolumn{1}{|c|}{ Escalas } & $X^{2}$ & $D f$ & $R M S E A$ & $(90 \%$ Cl) & SRMR & CFI & TLI \\
\hline 1 & Disposición al estudio & $25.862^{*}$ & 14 & .060 & $.020-.095$ & .041 & .952 & .928 \\
\hline 2 & Autoeficacia para la disposición al estudio & $22.041^{*}$ & 12 & .059 & $.014-.098$ & .047 & .966 & .940 \\
\hline 3 & Desempeño & $187.750^{*}$ & 117 & .050 & $.037-.063$ & .052 & .930 & .918 \\
\hline 4 & Atribuciones causales & $24.865^{*}$ & 19 & .036 & $.000-.071$ & .038 & .982 & .973 \\
\hline 5 & Autoevaluación del estudio y aprendizaje & $118.077^{*}$ & 119 & .047 & $.029-.064$ & .043 & .961 & .954 \\
\hline
\end{tabular}


En algunas escalas, los indicadores de ajuste relativo mostraron, en la primera solución, índices que no eran óptimos según la literatura; para re-especificar el modelo, se revisaron los índices de modificación (IM). En la escala de ejecución se encontraron pares de residuos entre el ítem 8 "Memorizo palabras clave para recordarme conceptos importantes" y el ítem 15 "Repito las ideas claves para memorizarlas" (IM=22.393), estos ítems aluden a las estrategias cognitivas de memorización que usa el estudiante al momento del estudio.

En la escala de autoeficacia para la disposición al estudio, se encontraron pares de residuos entre el ítem 4 "Establecer objetivos de estudio a largo plazo (mensual, semestral)" y el ítem 3 "Establecer objetivos de estudio a corto plazo (diario, semanal)" (IM=18.636), estos ítems aluden a la creencia del estudiante sobre su capacidad para establecer objetivos. En definitiva, las 5 escalas y sus respectivos ítems, luego del proceso de análisis factorial exploratorio y confirmatorio, quedó constituida de 53 ítems. Tres escalas tienen un formato de respuesta tipo Likert de 1 a 7 (ver tabla 4), y 2 escalas tienes un formato tipo Likert de 0 a 10 (ver tabla 5). Respecto de la consistencia interna de las escalas, esta mostró ser adecuada. El alfa de Cronbach fue en todos los casos superior a 0.6 al igual que el Omega de Mcdonald (Ver tabla 6).

Tabla 4. Escalas para medir las fases de autorregulación del aprendizaje en estudiantes de secundaria con escala de respuesta de 1 a 7

Escala de disposición al estudio

ítem En una escala de 1 a 7 donde 1 es nunca y 7 es siempre, responda: Con qué frecuencia, antes de empezar a estudiar o realizar una tarea académica:

$1 \quad$ Establezco objetivos académicos a corto plazo (diario, semanal)

$2 \quad$ Establezco objetivos académicos a largo plazo (mensual, semestral)

3 Ordeno los materiales para el estudio

$4 \quad$ Hago un horario para organizar mi tiempo de estudio

$5 \quad$ Planifico el tiempo que voy a dedicar a cada actividad

6 Tengo una lista con las tareas académicas por hacer

$\begin{array}{ll}7 & \text { Identifico que tareas académicas realizare primero }\end{array}$

\begin{tabular}{|l|l|l|l|l|l|l|}
\hline 1 & 2 & 3 & 4 & 5 & 6 & 7 \\
\hline 1 & 2 & 3 & 4 & 5 & 6 & 7 \\
\hline 1 & 2 & 3 & 4 & 5 & 6 & 7 \\
1 & 2 & 3 & 4 & 5 & 6 & 7 \\
\hline 1 & 2 & 3 & 4 & 5 & 6 & 7 \\
1 & 2 & 3 & 4 & 5 & 6 & 7 \\
\hline 1 & 2 & 3 & 4 & 5 & 6 & 7 \\
\hline
\end{tabular}

Escala de ejecución/desempeño (monitoreo, estrategias cognitivas y búsqueda de ayuda)

İtem Cuando estoy estudiando o realizando una tarea académica:

\begin{tabular}{|c|c|c|c|c|c|c|c|c|}
\hline 1 & Lo hago de acuerdo a un horario establecido por mí & 1 & 2 & 3 & 4 & 5 & 6 & 7 \\
\hline 2 & Evalúo si estoy aprendiendo durante el estudio & 1 & 2 & 3 & 4 & 5 & 6 & 7 \\
\hline 3 & Repaso los apuntes tomados en clases & 1 & 2 & 3 & 4 & 5 & 6 & 7 \\
\hline 4 & Tengo claro a que compañeros pedirle ayuda si fuera necesario & 1 & 2 & 3 & 4 & 5 & 6 & 7 \\
\hline 5 & Si no comprendo lo que leo, busco una forma para solucionarlo & 1 & 2 & 3 & 4 & 5 & 6 & 7 \\
\hline 6 & Sé dónde encontrar a personas que me puedan ayudar & 1 & 2 & 3 & 4 & 5 & 6 & 7 \\
\hline 7 & Identifico los contenidos que no entiendo bien & 1 & 2 & 3 & 4 & 5 & 6 & 7 \\
\hline 8 & Memorizo palabras clave para recordarme conceptos importantes & 1 & 2 & 3 & 4 & 5 & 6 & 7 \\
\hline 9 & Anoto las dudas para preguntar al profesor & 1 & 2 & 3 & 4 & 5 & 6 & 7 \\
\hline 10 & Cumplo con los objetivos de estudio & 1 & 2 & 3 & 4 & 5 & 6 & 7 \\
\hline 11 & Reviso el progreso de mi estudio & 1 & 2 & 3 & 4 & 5 & 6 & 7 \\
\hline 12 & Hago resúmenes de las ideas principales & 1 & 2 & 3 & 4 & 5 & 6 & 7 \\
\hline 13 & Reviso si mi planificación de estudio requiere modificación & 1 & 2 & 3 & 4 & 5 & 6 & 7 \\
\hline 14 & Reviso si mi procedimiento/estrategia de estudio es efectiva para aprender & 1 & 2 & 3 & 4 & 5 & 6 & 7 \\
\hline 15 & Repito las ideas claves para memorizarlas & 1 & 2 & 3 & 4 & 5 & 6 & 7 \\
\hline 16 & Si no estoy seguro de algún material o contenido pregunto a mis compañeros & 1 & 2 & 3 & 4 & 5 & 6 & 7 \\
\hline 17 & Cuando estudio reúno información de diferentes fuentes & 1 & 2 & 3 & 4 & 5 & 6 & 7 \\
\hline
\end{tabular}

Escala de autoevaluación de la planificación del estudio y el aprendizaje

\begin{tabular}{|c|c|c|c|c|c|c|c|c|}
\hline \multirow{2}{*}{ Ítem } & En una escala donde 1 es "nunca" y 7 es "siembre", responda. & \multirow{2}{*}{1} & \multirow{2}{*}{2} & \multirow{2}{*}{3} & \multirow{2}{*}{4} & \multirow{2}{*}{5} & \multirow{2}{*}{6} & \multirow{2}{*}{7} \\
\hline & Con qué frecuencia cuando termino mi estudio o una tarea académica reviso si: & & & & & & & \\
\hline 1 & Cumplí con mis objetivos propuestos & 1 & 2 & 3 & 4 & 5 & 6 & 7 \\
\hline 2 & Evalúo si completé mis desafíos personales de aprendizaje & 1 & 2 & 3 & 4 & 5 & 6 & 7 \\
\hline 3 & Realicé las tareas en los horarios establecidos & 1 & 2 & 3 & 4 & 5 & 6 & 7 \\
\hline 4 & Evalúo si alcancé las exigencias académicas establecidas por el profesor & 1 & 2 & 3 & 4 & 5 & 6 & 7 \\
\hline 5 & Completé mi lista de tareas académicas por hacer & 1 & 2 & 3 & 4 & 5 & 6 & 7 \\
\hline 6 & Reviso si logré los aprendizajes esperados por el profesor & 1 & 2 & 3 & 4 & 5 & 6 & 7 \\
\hline 7 & Mi planificación fue efectiva & 1 & 2 & 3 & 4 & 5 & 6 & 7 \\
\hline 8 & Reviso si logré mis objetivos personales de aprendizaje & 1 & 2 & 3 & 4 & 5 & 6 & 7 \\
\hline 9 & Terminé el estudio en el plazo planificado & 1 & 2 & 3 & 4 & 5 & 6 & 7 \\
\hline 10 & Reviso si comprendí los conceptos claves & 1 & 2 & 3 & 4 & 5 & 6 & 7 \\
\hline 11 & Identifico la estrategia que no me ayuda a aprender para no utilizarla en el futuro & 1 & 2 & 3 & 4 & 5 & 6 & 7 \\
\hline 12 & Evalúo si aprendí los contenidos centrales & 1 & 2 & 3 & 4 & 5 & 6 & 7 \\
\hline 13 & Requiero aumentar mi tiempo de estudio la próxima vez & 1 & 2 & 3 & 4 & 5 & 6 & 7 \\
\hline 14 & Reviso si avancé en relación con mi conocimiento previo & 1 & 2 & 3 & 4 & 5 & 6 & 7 \\
\hline
\end{tabular}


Tabla 5. Escalas para medir las fases de autorregulación del aprendizaje en estudiantes de secundaria con escala de respuesta de 0 a 10

Escala de Autoeficacia para la disposición al estudio

\begin{tabular}{|l|l|l|l|l|l|l|l|l|l|l|l|l|}
\hline \multirow{2}{*}{ Ítem } & En una escala donde cero es nada seguro y 10 es muy seguro responda. \\
\cline { 2 - 9 } & Antes de empezar a estudiar, en qué medida creo que es capaz de: & 0 & 1 & 2 & 3 & 4 & 5 & 6 & 7 & 8 & 9 & 10 \\
\hline 1 & Hacer un horario de estudio & 0 & 1 & 2 & 3 & 4 & 5 & 6 & 7 & 8 & 9 & 10 \\
\hline 2 & Tener una lista de tareas académicas por hacer & 0 & 1 & 2 & 3 & 4 & 5 & 6 & 7 & 8 & 9 & 10 \\
\hline 3 & Establecer objetivos de estudio a corto plazo (diario, semanal) & 0 & 1 & 2 & 3 & 4 & 5 & 6 & 7 & 8 & 9 & 10 \\
\hline 4 & Establecer objetivos de estudio a largo plazo (mensual, semestral) & 0 & 1 & 2 & 3 & 4 & 5 & 6 & 7 & 8 & 9 & 10 \\
\hline 5 & Elegir un lugar para estudiar sin distracciones & 0 & 1 & 2 & 3 & 4 & 5 & 6 & 7 & 8 & 9 & 10 \\
\hline 6 & Tener todos los materiales necesarios para estudiar & 0 & 1 & 2 & 3 & 4 & 5 & 6 & 7 & 8 & 9 & 10 \\
\hline 7 & Encontrar un lugar cómodo para estudiar (luz, to , ventilación) & &
\end{tabular}

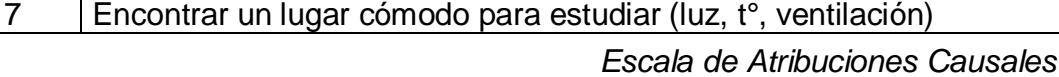

\begin{tabular}{|c|c|c|c|c|c|c|c|c|c|c|c|c|}
\hline \multirow{2}{*}{ Ítem } & \multicolumn{12}{|c|}{ En una escala donde creo es "no creo que sea asi" y 10 es "creo con mucha certeza que es asi", responda. } \\
\hline & En qué medida creo que la causa del fracaso en mi desempeño se debe a & & & & & & & & & & & \\
\hline 1 & La desmotivación del profesor & 0 & 1 & 2 & 3 & 4 & 5 & 6 & 7 & 8 & 9 & 10 \\
\hline 2 & Mi falta de esfuerzo en el estudio & 0 & 1 & 2 & 3 & 4 & 5 & 6 & 7 & 8 & 9 & 10 \\
\hline 3 & La falta de apoyo de mi familia & 0 & 1 & 2 & 3 & 4 & 5 & 6 & 7 & 8 & 9 & 10 \\
\hline 4 & La falta de apoyo de mis amigos & 0 & 1 & 2 & 3 & 4 & 5 & 6 & 7 & 8 & 9 & 10 \\
\hline 5 & La falta dedicación al estudio & 0 & 1 & 2 & 3 & 4 & 5 & 6 & 7 & 8 & 9 & 10 \\
\hline 6 & La despreocupación del profesor & 0 & 1 & 2 & 3 & 4 & 5 & 6 & 7 & 8 & 9 & 10 \\
\hline 7 & Que el profesor me tiene mala & 0 & 1 & 2 & 3 & 4 & 5 & 6 & 7 & 8 & 9 & 10 \\
\hline 8 & Mi desorganización del estudio & 0 & 1 & 2 & 3 & 4 & 5 & 6 & 7 & 8 & 9 & 10 \\
\hline
\end{tabular}

Tabla 6. Confiabilidad y número de ítems por cada factor de la batería

\begin{tabular}{|c|l|c|c|c|}
\hline \multicolumn{1}{|c|}{ Factores } & $\alpha$ & $\omega$ & $N^{\circ}$ ítems \\
\hline 1 & Estrategias de Disposición al Estudio & 0.764 & 0.764 & 7 \\
\hline 2 & Autoeficacia para la disposición al estudio & 0.792 & 0.794 & 7 \\
\hline 3 & Estrategias cognitivas y metacognitivas en el estudio y aprendizaje & 0.886 & 0.888 & 14 \\
\hline 4 & Búsqueda de ayuda & 0.635 & 0.635 & 3 \\
\hline 5 & Atribuciones causales de fracaso al esfuerzo y/o habilidad & 0.765 & 0.765 & 3 \\
\hline 6 & Atribuciones causales de fracaso a factores externos & 0.763 & 0.769 & 5 \\
\hline 7 & Autoevaluación del estudio y aprendizaje & 0.924 & 0.925 & 14 \\
\hline
\end{tabular}

\section{DISCUSIÓN}

El presente estudio tuvo como objetivo diseñar y estimar las propiedades psicométricas de escalas que miden las tres fases ARA en estudiantes secundarios ecuatorianos, con énfasis en dar respuesta a la medición de este constructo complejo. Los resultados ponen a disposición cinco escalas compuesta por un total de 53 ítems que representan la medición de la ARA a través de 7 procesos específicos. Los resultados presentados son consistente con resultados de estudios previos referidos al diseño de instrumentos para medir el multidimensional constructo de la autorregulación que han especificado seis subescalas (Toering et al., 2012). La ventaja de las escalas diseñadas en nuestro estudio es que la forma de aplicación es el uso a conveniencia; es decir, las escalas se pueden utilizar para medir el proceso de ARA en general y también puede utilizarse por separado, en la medición de un proceso específicos de ARA. Estos resultados reflejan un riguroso trabajo de diseño, verificable desde las evidencias sobre validez de contenido por medio de jueces expertos, validez de formato de respuesta por medio de la técnica de entrevista cognitiva, y de la estructura factorial por medio de un análisis factorial exploratorio.

Las implicancias de este estudio son psicométricas y educativas de alto valor. En cuanto a las implicancias psicométricas, existe evidencia teórica a favor del modelo propuesto, puesto que responde a la homologación de tres fases (disposición, desempeño y evaluación) de los distintos modelos existentes de ARA (Panadero, 2017a; Puustinen y Pulkkinen, 2001). Esta propuesta en particular realza la medición de este constructo completo desde un enfoque cognitivo motivacional del aprendizaje. Esto supone un abordaje holístico del desarrollo de habilidades, conocimientos, motivaciones, creencias y comportamientos por parte de los estudiantes en sus experiencias académicas, que van desde la planificación de una tarea académica hasta la persistencia frente a la frustración y ajuste respectivo de estrategias para lograr resultados exitosos. El constructo de AAR representa formas de aprendizaje académico autónomo, permanente y efectivo. Las implicaciones educativas versan sobre la capacidad de medir de manera parsimoniosa una variable -(AAR)muy relacionada con otros procesos psicoeducativos como el desempeño académico y el éxito académico de los estudiantes, la cual puede ser promovida por los profesores dentro del desarrollo cotidiano de sus asignaturas (Dignath y Büttner, 2018; Dent y Koenka, 2016). 
Con esta investigación se pone a disposición de la comunidad educativa e investigadores, escalas válidas y confiables para la medición de procesos psicológicos que son determinados por multi dimensiones, en este caso, el AAR. Se responde a la necesidad de contar con instrumentos que cumplan con propiedades psicométricas adecuadas, permite la evaluación de la efectividad o impacto de programas específicos implementados con el propósito de mejorar los niveles de ARA en sus diferentes fases y procesos en estudiantes de secundaria (López-Angulo et al., 2020). Así, este estudio se considera una contribución importante en el contexto de estudiantes de escuela secundaria en Ecuador, dado que no existía una escala válida y confiable que permitiera identificar qué nivel de desarrollo de los diferentes procesos de autorregulación presentan los estudiantes. Su uso permitirá mostrar mayor evidencia de sus características psicométricas y a su vez, permitirá identificar aquellas variables en niveles inadecuados de la ARA para, posteriormente, orientar el trabajo pedagógico en el fomento de esta competencia clave y crítica en el desempeño exitoso del estudiantado.

\section{CONCLUSIONES}

En base al objetivo general de esta investigación y a los elementos presentados en la discusión, se concluye: (1) las escalas para la medición de procesos de autorregulación del aprendizaje en estudiantes de secundaria ecuatorianos son 5 (tres unidimensionales y dos bidimensionales), y un total de 53 ítems, las que dan cuenta de las tres fases de ARA; (2) el análisis de cada escala mostró evidencias de adecuadas propiedades psicométricas tanto de evidencias de validez de contenido, de formato de respuesta, y de estructura factorial; (3) se pone a disposición escalas para la medición del proceso de ARA en general o la medición de procesos específicos en estudiantes de secundaria; y finalmente, (4) las escalas son útiles para investigadores que diseñan y evalúan el efecto de intervenciones como también para los profesores que buscan promover en sus estudiantes la autorregulación del aprendizaje.

\section{AGRADECIMIENTOS}

Al Proyecto FONDECYT de Iniciación en Investigación 2020 №11201054, titulado: "La relación recíproca entre la autorregulación del profesor y la autorregulación del aprendizaje y desempeño académico del estudiante. Un modelo explicativo en Educación Media"; y al proyecto INDIN 02/2019, titulado "Validación de un instrumento sobre variables predictoras de los docentes universitarios en la promoción intra curricular del aprendizaje autorregulado en sus estudiantes" financiado por la Dirección de Investigación de la Universidad Católica de la Santísima Concepción.

\section{REFERENCIAS}

Akhtar, J., y Mahmood, N., Development and Validation of an Academic Self-Regulation Scale for University students, https://doi.org/10.22251/jlcci.2019.19.15.1123, J. Behav. Sci., 23(2), 37-48, (2013).

Annalakshmi, N., Resilience and Academic Achievement among Rural Adolescents at-risk: Role of Self-regulation and Attachment Style, IJPP, ISSN: 2321-368X, 10(4), 260, (2019).

Ato, M., López, J., y Benavente, A., Un Sistema de Clasificación de los Diseños de Investigación en Psicología, https://doi.org/10.6018/analesps.29.3.178511, An. Psicol., 29(3), 1038-1059, (2013).

Cerezo, R., Bogarín, A., Esteban, M., y Romero, C., Process Mining for Self-regulated Learning Assessment in e-learning, https://doi.org/10.1007/s12528-019-09225-y, J Comput High Educ, 32(1), 74-88, (2020).

Cleary, T., The Development and Validation of the Self-Regulation Strategy Inventory-Self-Report, https://doi.org/10.1016/j.jsp.2006.05.002, J Sch Psychol, 44(1), 307-322, (2006).

Cleary, T., y Callan, G., Student Self-regulated Learning in an urban high school: Predictive Validity and Relations between Teacher Ratings and Student Self-reports, https://doi.org/10.1177/0734282913507653, J. Psychoeduc Assess, 32(4), 295-305, (2014).

Cleary, T. J., y Kitsantas, A., Motivation and Self-regulated Learning Influences on Middle School Mathematics Achievement, https://doi.org/10.17105/spr46-1.88-107, School Psych Rev, 46(1), 88-107, (2017).

Dent, A. L., y Koenka, A. C., The Relation between Self-regulated Learning and Academic Achievement across Childhood and Adolescence: A meta-analysis, https://doi.org/10.1007/s10648-015-9320-8, Educ Psychol Rev., 28(3), 425-474, (2016).

DiFrancesca, D., Nietfeld, J., y Cao, L., A Comparison of High and Low Achieving Students on Self-regulated Learning Variables, https://doi.org/10.1016/j.lindif.2015.11.010, Learn Individ Differ, 45, 228-236, (2016).

Dignath, C., y Büttner, G., Teachers' Direct and Indirect Promotion of Self-regulated Learning in Primary and Secondary School Mathematics Classes-insights from video-based classroom observations and teacher interviews, https://doi.org/10.1007/s11409-018-9181-x, Metacogn Learn, 13(2), 127-157, (2018). 
El-Adl, A., y Alkharusi, H., Relationships between Self-regulated Learning Strategies, Learning Motivation and Mathematics Achievement, https://doi.org/10.18844/cjes.v15i1.4461, Cypriot J. Educ. Sci., 15(1), 104-111, (2020).

Fernandez-Rio, J., Cecchini, J., y otros tres autores, Self-regulation, Cooperative Learning, and Academic Self-efficacy: Interactions to Prevent School Failure, https://doi.org/10.3389/fpsyg.2017.00022, Front. Psychol., 8(1), 1-10, (2017).

Hair, J. F., Black, W. C., Babin, B. J., y Anderson, R. E., Multivariate Data Analysis: Pearson new international edition, (2014).

Holgado-Tello, F., Chacón-Moscoso, S., Barbero-García, I., y Vila-Abad, E., Polychoric versus Pearson Correlations in Exploratory and Confirmatory Factor Analysis of Ordinal Variables, https://doi.org/10.1007/s11135-008-9190-y, Qual Quant, 44(1), 153-166, (2010).

Izquierdo, I., Olea, J., y Abad, F., El Análisis Factorial Exploratorio en Estudios de Validación: Usos y recomendaciones, https://doi.org/10.7334/psicothema2013.349, Psicothema, 26(3), 395-400, (2014).

Jansen, R., Van Leeuwen, A., y otros tres autores, Validation of the Self-regulated Online Learning Questionnaire, https://doi.org/10.1007/978-3-319-98572-5_9, J. Comput High Educ, 29(1), 6-27, (2017).

Kline, R. B., Principles and Practice of Structural Equation Modeling, $3^{\text {rd }}$ edition, The Guilford Press, New York, NY, (2011).

Lawrence, A., y Saileella, K., Self-Regulation of Higher Secondary Students in Relation to Achievement in Mathematics, Int. Multidiscip. Res. J, ISSN: 2231-5780, 9(1), 258-265, (2019).

León-Ron, V., Sáez, F., y otros cuatro autores, Revisión Sistemática sobre Instrumentos de Autorregulación del Aprendizaje diseñados para Estudiantes, Rev. Espacios, ISSN: 0798-1015, 41(11), 1-9, (2020).

López-Angulo, Y., Sáez-Delgado, F., Arias-Roa, N., y Díaz-Mujica, A., Revisión Sistemática sobre Instrumentos de Autorregulación del Aprendizaje en Estudiantes de Educación Secundaria, Inf. Tecnol., ISSN: 0716-8756, 31(4), (2020).

Panadero, E., A Review of Self-regulated Learning: Six Models and Four Directions for Research, https://doi.org/10.3389/fpsyg.2017.00422, Front. Psychol, 8, 1-28, (2017).

Pintrich, P., Smith, D., García, T., y McKeachie, W., A., Manual for the Use of the Learning Questionnaire Motivated Strategies for (MSLQ), https://doi.org/10.5901/mjss.2015.v6n1p156, Mediter J Soc Sci, 6(1), 156-164, (1991).

Puustinen, M., y Pulkkinen, L., Models of Self-regulated Learning: a Review, https://doi.org/10.1080/00313830120074206, Scan J Educ Res, 45(3), 269-286, (2001).

Sáez, F., Bustos, C., y Díaz, A., Autoeficacia Cuestionario de Autorregulación de Estudio Readiness, https://doi.org/10.15689/ap.2017.1701.10.13348, Aval. Psicol, 17(1), 92-100, (2018).

Sáez, F., Mella, J., y López, Y., Atribuciones Causales un Factor a Considerar en la Comprensión del Abandono en Educación Superior: Instrumento para su Medición. Congresos CLABES, 179-187, (2019).

Şen, Ş., y Yilmaz, A., Devising a Structural Equation Model of Relationships between Preservice Teachers' Time and Study Environment Management, Effort Regulation, Self-efficacy, Control of Learning Beliefs, and Metacognitive Self-regulation, Sci. Educ. Int., ISSN: 2077-2327, 27(2), 301-316, (2016).

Toering, T., Elferink-Gemser, M., y otros tres autores, Measuring Self-regulation in a Learning Context: Reliability and Validity of the Self-Regulation of Learning Self-Report Scale (SRL-SRS), https://doi.org/10.1080/1612197X.2012.645132, Int J Sport Exerc Psychol, 10(1), 24-38, (2012).

Urrutia, M., Barrios, S., Gutiérrez, M., y Mayorga, M., Métodos Óptimos para Determinar Validez de Contenido, Rev. Cub. Educ. Méd. Super., ISSN: 1561-2902, 28(3), 547-558, (2015).

Yan, Z., Self-assessment in the Process of Self-regulated Learning and its Relationship with Academic Achievement, https://doi.org/10.1080/02602938.2019.1629390, Assess Eval High Educ, 45(2), 224-238, (2020).

Zimmerman, B., Attaining Self-regulation a Social Cognitive Perspective, Handbook of Self-Regulation, 13-40. Academic Press, San Diego, Estados Unidos (2000). 